\title{
Joint Load Balancing and Power Allocation for Hybrid VLC/RF Networks
}

\author{
Mohanad Obeed*, Anas M. Salhab*, Salam A. Zummo*, and Mohamed-Slim Alouini ${ }^{+}$ \\ *King Fahd University of Petroleum \& Minerals, Dhahran, Eastern Province, Saudi Arabia \\ Email: $\{\mathrm{g} 201106250$, salhab, zummo $@$ @kfupm.edu.sa \\ ${ }^{+}$King Abdullah University of Science and Technology, Thuwal, Makkah Province, Saudi Arabia \\ Email: slim.alouini@kaust.edu.sa
}

\begin{abstract}
In this paper, we propose and study a new joint load balancing (LB) and power allocation (PA) scheme for a hybrid visible light communication (VLC) and radio frequency (RF) system consisting of one RF access point (AP) and multiple VLC APs. An iterative algorithm is proposed to distribute the users on the APs and distribute the powers of these APs on their users. In PA subproblem, an optimization problem is formulated to allocate the power of each AP to the connected users for the total achievable data rates maximization. It is proved that the PA optimization problem is concave but not easy to tackle. Therefore, we provide a new algorithm to obtain the optimal dual variables after formulating them in terms of each other. Then, the users that are connected to the overloaded APs and receive less data rates start seeking for other APs that offer higher data rates. Users with lower data rates continue re-connecting from AP to other to balance the load only if this travel increases the summation of the achievable data rates and enhances the system fairness. The numerical results demonstrate that the proposed algorithms improve the system capacity and system fairness with fast convergence.
\end{abstract}

Index Terms-Hybrid visible light communication and radio frequency (VLC/RF), power allocation, load balancing.

\section{INTRODUCTION}

The growing demand for high data rate and licencefree spectrum applications has stimulated recently many researchers to investigate the visible light communication (VLC) as a promising technique for indoor communication. As known, the radio frequency (RF) communications are becoming more restricted because of the limited spectrum resources in wireless networks. As a solution, the VLC has been introduced in indoor environment to overcome the RF limitations and provide better services to the users.

The VLC is a communication system that uses light emitting diode (LED), which is highly energy efficient, as a transmitter to emit both the light and information signals to the users. The receiver must be equipped with a photo detector (PD) device that converts the optical signal to an electrical signal. Hence, the data is transmitted using an intensity modulation (IM) technique at the transmitter and received using a direct detection (DD) technique at the receiver (IM/DD). In addition for being license-free and secure systems, the VLCs are known for their ability to reject RF interference and their high bandwidth potential [1]. Unlike the RF network, the VLC works properly only when the line of site (LoS) component between the transmitter and receiver is available. Therefore, supplementing the VLC network with a RF AP increases the network coverage and improves the total system capacity [2].
Authors of [3] considered the cooperation between two VLC APs to increase the rate region by controlling the transmission power. In [4], the authors studied the advantages of combining the RF and VLC and showed that the users are distributed dynamically on both the VLC and RF networks based on the user channel condition. Then, the users with lower data rates are transferred to the RF AP to increase their data rates. To decrease the number of handovers, [5] proposed a dynamic load balancing (LB) scheme where the quasi-static users are connected to VLC APs, while the unfixed users are connected to RF AP. In [6], power and bandwidth allocation was investigated for a system consisting of only one VLC AP and one RF AP, which is easy to tackle due to the absence of interference. To study the joint load balancing and resource allocation, [7] proposed a bidirectional allocation game where at first, every user is serviced from the AP that provides him with the highest signal-to-noise ratio (SNR) with time resource allocation. The users that receive a poor quality of service (QoS) are then reconnected to a neighboring AP only in case that it can provide higher QoS. The resource allocation and load balancing operations are implemented iteratively, until all users are satisfied or have their own optimal APs.

Up to the authors knowledge, the joint LB and power allocation (PA) for a hybrid VLC/RF network has not been presented or studied before. In this paper, we study the two problems of PA and LB in a hybrid VLC/RF network for the sake of data rate maximization and system fairness improvement. The network consists of multiple VLC APs and one RF AP. First, every user is connected to its nearest AP. Then, each AP allocates the power for the associated users in order to maximize the summation of the achievable data rates per AP. After that, the users with the lower data rates start reconnecting from AP to other to balance the load only in case this transfer increases the summation of the achievable data rates. This transfer of users continues until no improvement in the summation of data rates is achieved. In the PA optimization subproblem, we formulate the problem for maximizing the summation of the achievable users data rates under certain QoS constraints. These QoS constraints are formulated to control the tradeoff between the system capacity and system fairness. For a given interference information, the PA problem is proved to be concave but not easy to tackle. Similar PA problems were studied before such as [6] and [8]. In finding the dual variables, the authors used the gradient 
decent algorithm, which is very sensitive to step size selection and needs a large number of iterations for convergence. Here, we derive a more efficient algorithms to find the dual variables after formulating them in terms of each other. The inter-cell interference makes the joint PA and LB problem very difficult. Therefore, an iterative algorithm is proposed to assure that the power is allocated to the users based on the exact interference information. This work is different from the work of [7] in several aspects as follows: 1) we generalize the system to include a RF AP, 2) in the PA subproblem, the authors in [7] performed a time resource allocation problem, while we allocate the power for the users, and 3) the PA problem is formulated as a mathematical optimization problem and not by using the fuzzy logic as in [7].

The rest of this paper is organized as follows. The system and channel models are introduced in Section II. In Section III, we present the problem formulation and proposed algorithms. Some simulation results are shown and discussed in Section IV. Ultimately, the paper is concluded in Section V.

\section{SySTEM AND CHANNEL MODELS}

The system under consideration consists of $N_{a p}$ VLC APs, one RF AP, and $N_{u}$ users as shown in Fig. 1. The users are distributed uniformly in the room and the APs are fixed in the ceiling of the room. Each VLC AP is equipped with multiple LEDs that use IM to transmit the light signal to the users, which receive the light by a PD. The RF AP is assumed to cover all the room area. Also, the location of users are assumed to be unchanged during a short time $T$. Thus, the VLC and RF channel-state-information (CSI) of both the VLC and RF links are considered to be constant during this period. We assume that the maximum available bandwidth at the AP $i$ is divided fairly among all the users connected to that AP.

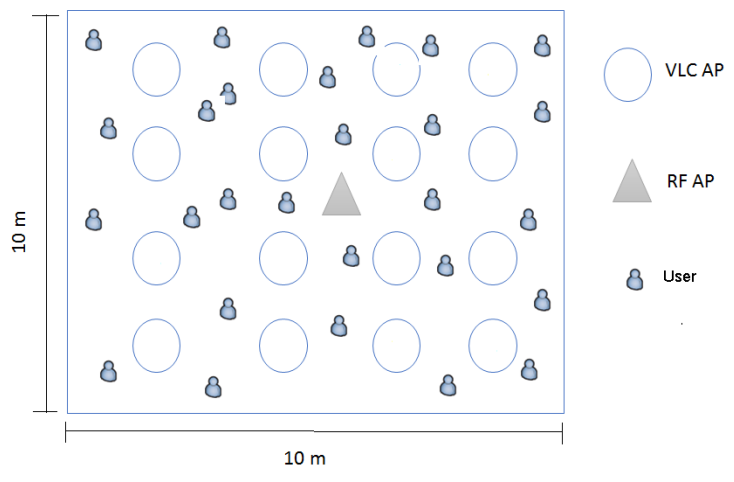

Fig. 1: System model.

\section{A. VLC Channel}

The LoS VLC channel between the $i^{t h}$ LED and the $j^{t h}$ user can be expressed as follows [9]

$$
h_{i, j}^{(v)}=\frac{(m+1) A_{p}}{2 \pi d_{i, j}^{2}} \cos ^{m}(\phi) g_{o f} f(\theta) \cos (\theta),
$$

where $m$ is the Lambertian index that is given by $m=$ $-1 / \log _{2}\left(\cos \left(\theta_{1 / 2}\right)\right.$, where $\theta_{1 / 2}$ is the half intensity radiation angle, $A_{p}$ is the physical area of the PD, $d_{i, j}$ is the distance between the $i^{t h}$ AP to the $j^{t h}$ user, $g_{o f}$ is the gain of the optical filter, $\phi$ is the angle of radiance at the AP, $\theta$ is the angle of incidence at the PD, and $f(\theta)$ is the optical concentrator gain, which is a function of $\theta$ that is given by $f(\theta)=\frac{n^{2}}{\sin ^{2}(\Theta)}$ if $\theta \leq \Theta$ or 0 if $\theta>\Theta$, where $n$ is the refractive index and $\Theta$ is the semi-angle of the field-of-view (FoV) of PD. In a VLC network, the LED has to operate in the linear region so that the optical power at its output is a linear function of the input voltage. The non-LoS VLC transmissions are assumed to be unsuccessful so that we work only on LoS paths. This assumption does not affect the proposed algorithm since it does not depend on a specific channel model. Therefore, when the LoS path is available, $h_{i, j}^{(v)}$ is given by (1); otherwise, $h_{i, j}^{(v)}=0$. The probability of the availability of the LoS from an AP to a user is denoted by $\alpha$ and it is assumed to be uniformly distributed. It was shown in [6] that the average electrical power of the received signal at user $j$ from the VLC AP $i$ is calculated as follows

$$
P_{\text {elec }, j, i}=\left(\rho \sqrt{P_{j, i}^{(v)}} h_{j, i}^{(v)}\right)^{2}
$$

where $\rho$ is the efficiency of converting the light to the electrical signal, $P_{j, i}^{(v)}$ is the allocated power for user $j$ from the AP $i$, where $P_{i, \max }^{(v)}$ is the maximum transmitted optical power of the AP $i$. The received SNR at user $j$ from the VLC AP $i$ can be expressed as

$$
\Gamma_{j, i}^{(v)}=\frac{\left(\rho \sqrt{P_{j, i}^{(v)}} h_{j, i}^{(v)}\right)^{2}}{\left(B_{i, \max }^{(v)} / N_{i}\right) N_{0}^{(v)}+\rho^{2} \sum_{l=1, l \neq i}^{N_{p}} P_{B_{j, l}}^{(v)} h_{j, l}^{(v)_{2}}},
$$

where $B_{i, \max }^{(v)}$ is the maximum available bandwidth at the AP $i, N_{i}$ is the number of users connected to the AP $i$, $N_{0}^{(v)}$ is the noise power spectral density, and $P_{B_{j}, l}^{(v)}$ is the interference power at user $j$ caused by the AP $l$. It is worth to note that $P_{B_{j, l}}^{(v)}$ must be calculated carefully since it represents the power allocated from the AP $l$ to the same frequency spectrum that is allocated for user $j$. For instance, if the AP $l$ is associated with 4 users, and user $j$ along with another user are connected to AP $i, P_{B_{j}, l}^{(v)}$ should be calculated as the summation of the powers of 2 users out of the 4 users that are connected to the AP $l$ and use the same spectrum frequency used by user $j$.

Here, Shannon's capacity formula is used to express the achievable data rate. This formula measures the limit of the performance regardless the kinds of the coding and modulation techniques. This formula is employed due to its mathematical tractability since using different modulation schemes does not affect significantly the optimality of the achieved data rate [10]. Hence, the upper limit of the data rate that can be achieved at user $j$ from the VLC AP $i$ is given by

$$
R_{j . i}^{(v)}=\left(B_{i, \max }^{(v)} / N_{i}\right) \log _{2}\left(1+\Gamma_{j, i}^{(v)}\right) .
$$

\section{B. RF Channel}

As mentioned before, the RF cell is assumed to cover all the room area and a spectrum of a total bandwidth $B_{\max }^{(r)}$ at the RF $\mathrm{AP}$ is considered to be flat fading and can be partitioned into non-overlapping channels of unequal bandwidths. According 
to [2], the RF channel between user $j$ and the RF AP is expressed as

$$
h_{j}^{(r)}=\sqrt{10^{-\frac{L(d)}{10}}}\left(\sqrt{\frac{I}{I+1}} h_{d}+\sqrt{\frac{1}{I+1}} h_{s}\right),
$$

where $I$ is the Rician component which is equal to $10 \mathrm{~dB}$ for indoor $60 \mathrm{GHz}$ mmWave, $h_{d}=\sqrt{0.5}(1+j)$ is the LoS fading channel, $h_{s}$ is a circular normal distributed with zero mean and unity variance and it is the fading channel of the non-LoS direction, and $L(d)$ is a function of distance $d$ and it is given by

$$
L(d)=L\left(d_{0}\right)+10 v \log _{10}\left(d / d_{0}\right)+Z,
$$

where $L\left(d_{0}\right)=68 \mathrm{~dB}$ is the reference path loss at some reference distance $d_{0}=1 \mathrm{~m}, v=1.6$ is the path loss exponent, and $Z$ is assumed to be $\mathcal{N}(0,3.6 \mathrm{~dB})$ and it is defined as the shadowing component. The SNR at the user $j$ from the RF AP is given by

$$
\Gamma_{j}^{(r)}=\frac{P_{j}^{(r)}\left|h_{j}^{(r)}\right|^{2}}{\left(B_{\max }^{(r)} / N_{r}\right) N_{0}^{(r)}}
$$

where $B_{\max }^{(r)}$ is the maximum available bandwidth at the RF AP and $N_{r}$ is the number of users connected to the RF AP. The achievable data rate that achieved by user $j$ from the RF AP is given by

$$
R_{j}^{(r)}=\left(B_{\max }^{(r)} / N_{r}\right) \log _{2}\left(1+\Gamma_{j}^{(r)}\right) .
$$

\section{PRoblem Formulation}

The problem here is how to assign all the users to the APs and how to allocate the APs powers for the assigned users aiming to maximize the system capacity with keeping the fairness between the users at an acceptable level. Therefore, we start by assigning the users based on their distance from the APs, where each user selects the closest AP to it. Then, each AP performs its own power allocation problem for its associated users. The achievable data rate of user $j$ is written as

$$
R_{j, i}= \begin{cases}R_{j, i}^{(v)}, & i \in \mathcal{C}_{v} \\ R_{j, i}^{(r)}, & i \in \mathcal{C}_{r}\end{cases}
$$

where $\mathcal{C}_{v}$ denotes the set of the VLC APs and $\mathcal{C}_{r}$ denotes the RF AP.

\section{A. Power Allocation in VLC and RF Access Points}

Our goal in this section is to allocate the power for the $N_{i}$ users that are connected to the AP $i$. The objective function here is to maximize the summation of the users capacity under certain QoS constraints. These constraints are formulated to guarantee some fairness for users per APs. We formulate the optimization problem as a general form for the VLC APs or the RF AP. Hence, $\left[\begin{array}{llll}P_{j, i} & B_{j, i} & h_{j, i} & N_{0}\end{array}\right]=$ $\left[\begin{array}{llll}P_{j, i}^{(v)} & \left(B_{i, \max }^{(v)} / N_{i}\right) & \rho h_{j, i}^{(v)} & N_{0}^{(v)}\end{array}\right]$, if the AP $i$ is a VLC $\mathrm{AP}$, and if the AP $i$ is a RF AP, so $\left[\begin{array}{llll}P_{j, i} & B_{j, i} & h_{j, i} & N_{0}\end{array}\right]=$ $\left[\begin{array}{llll}P_{j}^{(r)} & \left(B_{\max }^{(r)} / N_{r}\right) & h_{j}^{(r)} & N_{0}^{(r)}\end{array}\right]$. Therefore, the optimization problem of the AP $i$ can be written as

$$
\begin{array}{cl}
\max _{P_{1, i}, \ldots, P_{N_{i}, i}} & \sum_{j=1}^{N_{i}} R_{j, i} \\
\text { s.t. } & R_{j, i} \geq \gamma_{j, i}, j=1, \ldots, N_{i} \\
& \sum_{j=1}^{N_{i}} P_{j, i} \leq P_{i, \max }, \\
& P_{j, i} \geq 0 \quad \forall j,
\end{array}
$$

where $N_{i}$ is the number of users connected to the AP $i$ and $\gamma_{j, i}$ is the minimum data rate that can be achieved at user $j$ from the AP $i$, which given by

$$
\gamma_{j, i}=\beta \frac{B_{i, \max }}{N_{i}} \log \left(1+\frac{\left(P_{i, \max } / N_{i}\right)\left|h_{j, i}\right|^{2}}{\left(B_{i, \max } / N_{i}\right) N_{0}+X_{i, j}}\right),
$$

where $X_{i, j}$ is the interference term of user $j$ and is equal to $\rho^{2} \sum_{l=1, l \neq i}^{N_{p}} P_{B_{j, l}}^{(v)} h_{j, l}^{(v) 2}$ if the AP $i$ is VLC, and in the case of RF AP, $X_{i, j}=0$, and $\beta$ is a value in the interval $[0,1]$. If we select $\beta=1$, this means that the AP will distribute its resources fairly (regardless of users channels), where each user gains the same power $P_{i, \max } / N_{i}$ and same bandwidth $B_{i, \max } / N_{i}$. On the other hand, if $\beta$ is close to zero, this means that the objective function is released from the first constraint, which leads to increase the AP capacity. Jain's fairness index is used to measure the AP fairness and the whole system fairness. Since the fairness of the AP $i$ is given by

$$
F_{i}=\frac{\left(\sum_{j=1}^{N_{i}} R_{j, i}\right)^{2}}{N_{i} \sum_{j=1}^{N_{i}} R_{j, i}^{2}}
$$

and the fairness of the system is given by

$$
F_{s}=\frac{\left(\sum_{i=1}^{N_{a p}} \sum_{j=1}^{N_{i}} R_{j, i}\right)^{2}}{N_{a p} \sum_{i=1}^{N_{a p}} \sum_{j=1}^{N_{i}} R_{j, i}^{2}} .
$$

The Problem in (10) is not easy to tackle since in the case where the AP $i$ is a VLC AP, the interference term in the objective function makes the problem difficult. On the other hand, if the interference terms are given, the Problem in (10) becomes a concave problem and can be solved by using the Lagrangian dual problem. First, we solve this problem under the assumption that the interference terms are given then we provide an iterative algorithm that provides the exact interference information and achieves the optimal power allocation. Obviously, the Constraints in (10c) and (10d) are linear functions, while the objective function and the Constraint in (10b) are well known as concave functions as shown in [6] and [8]. Therefore, the optimization Problem in (10) is a concave problem with one global optimum solution. Hence, we can use the dual problem to achieve the optimal solution, where the strong duality holds in the concave problems. The dual optimization problem of the Problem in (10) can be expressed as follows

$\zeta=-\sum_{j=1}^{N_{i}} R_{j, i}-\sum_{j=1}^{N_{i}} \mu_{j, i}\left(R_{j, i}-\gamma_{j, i}\right)+v_{i}\left(\sum_{j=1}^{N_{i}} P_{j, i}-P_{i, \max }\right)$,

where $\mu_{j, i} \geq 0, \forall j \in N_{i}$, and $v_{i} \geq 0$ are the the Lagrangian dual variables. 
In the following, a new efficient algorithm is proposed to solve Problem in (10) by minimizing the dual Problem in (14). From the Karush-Kuhn-Tucker (KKT) conditions [11], we have

$$
\frac{\partial \zeta}{\partial P_{j, i}}=0
$$

where

$$
P_{j, i}=\frac{\left(1+\mu_{j, i}\right) B_{j, i}}{v_{i} \ln (2)}-\frac{N_{0} B_{j, i}+X_{i, j}}{\left|h_{j, i}\right|^{2}},
$$

where the variable $\mu_{j, i}$ must guarantee that the Constraint in (10b) is feasible and $v_{i}$ must guarantee that the Constraint in (10c) is feasible too. Now, these dual variables must be found to obtain $P_{j, i} \forall j$. In [6] and [8], the authors found such dual variables by using the subgradient method, which is an iterative algorithm that needs a large number of iterations to converge and a very careful selection of the step size. Here, we find a closed-form expression for $v_{i}$ in terms of $\mu_{j, i}$ and vise versa. Then, we solve them alternatively until they converge. First, it is trivial showing that the Constraints in (10c) must hold with equality at optimality. Otherwise, we can increase one of the power variables until the constraints hold with equality, which leads to increasing the objective function, and hence, contradicting the optimality. By substituting (16) in (10c), we have

$$
v_{i}=\frac{\sum_{j=1}^{N_{i}} B_{j, i}\left(1+\mu_{j, i}\right)}{\ln (2)\left(P_{i, \max }+\sum_{j=1}^{N_{i}} \frac{B_{j, i} N_{0}+X_{i, j}}{\left|h_{j, i}\right|^{2}}\right)} .
$$

Similarly, by substituting (16) in (10b), $\mu_{j, i}$ must be

$$
\mu_{j, i} \geq \frac{v_{i} \ln (2) P_{j, i}^{\min }}{B_{j, i}}+\frac{v_{i} \ln (2)\left(B_{j, i} N_{0}+X_{i, j}\right)}{B_{j, i}\left|h_{j, i}\right|^{2}}-1,
$$

where $P_{j, i}^{\min }$ is given by

$$
P_{j, i}^{\min }=\frac{\left(2^{\left(\gamma_{j, i} / B_{j . i}\right)}-1\right)\left(B_{j, i} N_{0}+X_{i, j}\right)}{\left|h_{j, i}\right|^{2}} .
$$

Hence, (17) and (18) depend on each other and can be solved alternatively starting from an initial value of one of them until they converge. After that, (16) is used to find the optimal powers for the assigned users. We propose Algorithm 1 to solve the optimization problem in (10) with a given interference information. Now, in the VLC APs, the problem

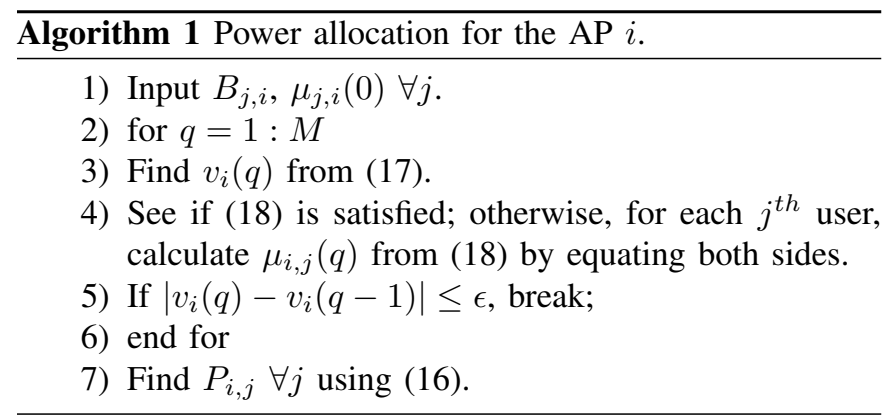

is how to find the instantaneous interference $\left(X_{j, i}\right)$ of each user, which is difficult to be found because the term $X_{j, i}$ of user $j$ depends on all the powers of the APs that are allocated for the $j^{\text {th }}$ user frequency spectrum. Therefore, we provide an iterative algorithm that solves the PA problem of all the VLC APs with finding the instantaneous interference of each user. Algorithm 2 provides the optimal power allocation for

Algorithm 2 Allocating the power for all the VLC APs with a given distributed users.

1) Each AP allocates the power for the users equally.

2) for $q=1: M$

3) Calculate $X_{i, j}^{(q)}$ for all users in the system.

4) Perform Algorithm 1 for all APs.

5) if $\sum_{i=1}^{N_{p}} \sum_{j=1}^{N_{i}}\left(X_{i, j}^{(q)}-X_{i, j}^{(q-1)}\right)^{2} \leq \epsilon$; break;

6) End for

a given distributed users. It is worth stating that the RF AP performs its power allocation using Algorithm 1 and it is not included in Algorithm 2. This is because the interference of the users connected to the RF AP is zero.

\section{B. Load Balancing}

As stated earlier, initially, each user is connected to its closest AP. Then, each AP performs its own power allocation problem as shown in the previous section. However, some APs will be overloaded, which may cause some users connected to these APs to receive a poor QoS. Therefore, after the PA optimization problem (Algorithm 2) is performed, the user with the poorest QoS is reconnected to either the RF AP or another adjacent VLC AP if the later can provide a better service and increase the system capacity. The users connected to the RF AP do not receive any interference so that the strongest candidate AP for the user, which needs to reconnect to another AP is the RF AP. The approach here is to arrange the users as a queue starting from the user with the lowest QoS up to the highest quality serviced user. Then, each user in its turn tests if the RF AP can provide a better achievable data rate for it or not. If so, the user migrates to the RF AP; otherwise, the user transfers to another adjacent AP if that AP can provide a better achievable data rate for him. These procedures continue until no improvement in the system capacity can be achieved. From the PA Problem in (10), each VLC AP offers for each user its QoS denoted by $\gamma_{j, i}$, which is a function of the number of users connected to that AP as shown in (11). Hence, the maximum offered achievable data rate for user $j$ that is connected to the AP $i$ is given by

$$
\bar{R}_{j, k}=\max _{k \in \chi_{i}}\left(\gamma_{j, k}\right)
$$

where $\chi_{i}$ is the set of the APs that are very close to the AP $i$ and $N_{k}<N_{i}-1 \forall k$. Since $\gamma_{j, k}$ depends on the value of $\beta$, if $N_{k}=0$, that means the AP $k$ offers all its resources for the coming user, and hence, $\beta=1$ in (20). Otherwise; if $N_{k} \geq 1, \beta$ is determined by the AP $k$. It is important to state that the transfer of a user connection changes the interference information, which enforces the system to reimplement Algorithm 2 with each transfer. This process of transfer continues only if there is an improvement in the system capacity. 
TABLE I: Simulation Parameters

\begin{tabular}{lc}
\hline Name of the Parameters & Value of the Parameters \\
\hline Room hight & $3 \mathrm{~m}$ \\
Maximum bandwidth of VLC AP, $B_{\max }^{(v)}$ & $30 \mathrm{MHz}$ \\
Maximum bandwidth of RF AP, $B_{\max }^{(r)}$ & $30 \mathrm{MHz}$ \\
The physical area of a PD, $A_{p}$ & $0.1 \mathrm{~cm}^{2}$ \\
Half-intensity radiation angle, $\theta_{1 / 2}$ & $60^{\circ}$ \\
FoV semi-angle of PD, $\Theta$ & $90^{\circ}$ \\
Gain of optical filter, $g_{o f}$ & 1 \\
Refractive index, $n$ & 1.5 \\
Efficiency of converting optical to electric, $\rho$ & 1 \\
Transmit power of the VLC AP, $P_{\max }^{(v)}$ & $4 \mathrm{watt}$ \\
Transmit power of the RF AP, $P_{\max }^{(r)}$ & $2 \mathrm{watt}$ \\
Noise power spectral density of LiFi, $N_{0}^{(v)}$ & $10^{-21} \mathrm{~A}^{2} / \mathrm{Hz}$ \\
Variance of AWGN in RF AP, $N_{0}^{(r)}$ & $10^{-19} \mathrm{~A}^{2} / \mathrm{Hz}$ \\
\hline
\end{tabular}

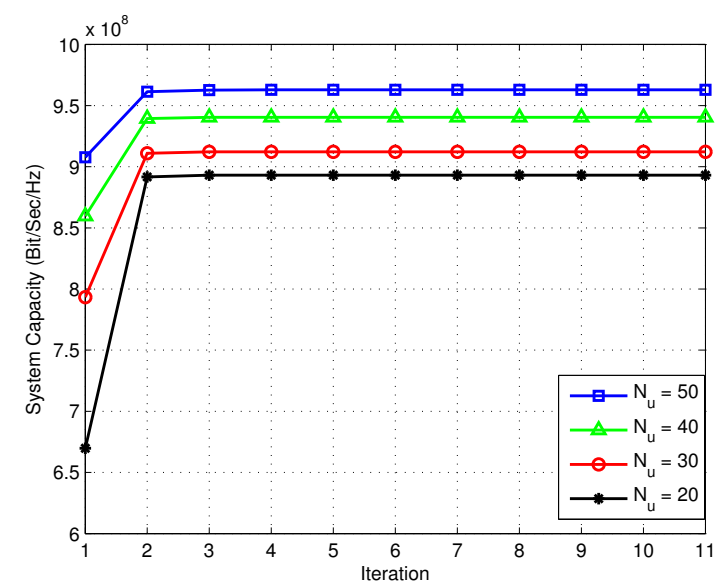

Fig. 2: System capacity versus number of iterations for Algorithm 2 with different numbers of system users.

\section{Simulation Results}

In the following, we verify the capability of the proposed algorithms for enhancing the performance of the hybrid VLC/RF network. We show the convergence of the proposed algorithms and how they increase the system capacity. A $10 \times 10$ room area is assumed with 16 VLC APs and 1 RF AP fixed in the ceiling. The values of all parameters in the considered VLC/RF system are given in Table I. MonteCarlo simulation is used to asses the performance of the proposed algorithms where in each simulation iteration, a uniform random number is generated between 0 and 1. If that number is less than $\alpha$ (the probability of the availability of the LoS), the LoS component is available; otherwise, it is not available.

Fig. 2 shows the convergence of Algorithm 2. It is clear that Algorithms 2 needs at most three iterations to converge. Furthermore, the number of iterations needed to converge does not depend on the number of users in the system, which means that the number of users does not affect the convergence of Algorithm 2. This indicates that Algorithm 2 rapidly converges to the optimal solution.

In Fig. 3, the relation between the system capacity and number of reconnected users is shown with different numbers of total users. It can be seen that the more the users transfers, the better the system capacity till the system saturates. Also, its obvious that more users transfers are needed for the system

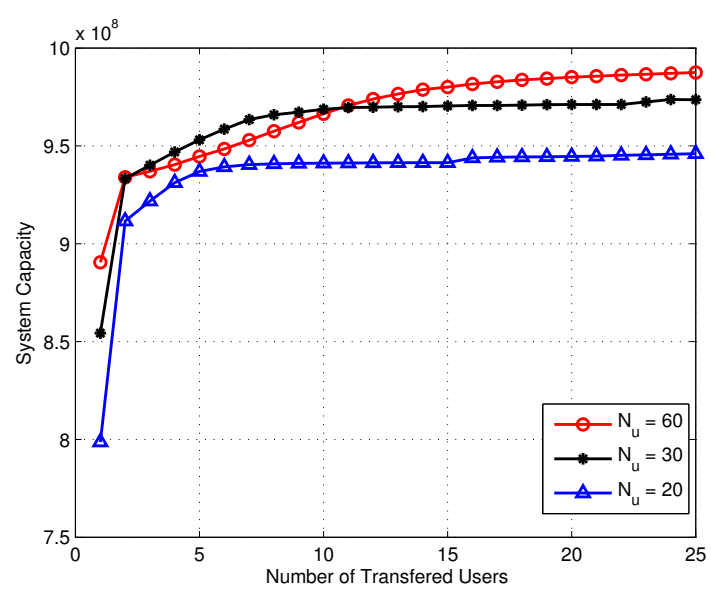

Fig. 3: System capacity against the number of the transferred users for different numbers of system users.



Fig. 4: System fairness against the number of the transferred users for different numbers of system users.

to reach the saturation point as the number of users increases, as expected. In other words, the rate of increasing the system capacity with 60 users is less than the rate of increasing the system capacity with 20 or 40 users.

The impact of total number of users on the system fairness is studied in Fig. 4. It is clear from this figure that as the number of transferred users increases, the system fairness is more enhanced with the best results achieved at the minimum number of total users. Enhancing the system fairness and capacity together with each user transfer comes from the fact that the users with poor services travel from the overloaded APs to have better services from other less overloaded APs. This helps in decreasing the variance of the received data rate among users and provides more efficient utilization.

Fig. 5 shows the relation between the number of users and the total system capacity for different values of $\alpha$. We also investigate the effect of number of users on the system fairness in Fig. 6. As can be seen from Fig. 5 and Fig. 6, as the probability of the LoS availability decreases, the system capacity increases, while the fairness decreases. This is because increasing the blockages rate in the hybrid VLC/RF systems 


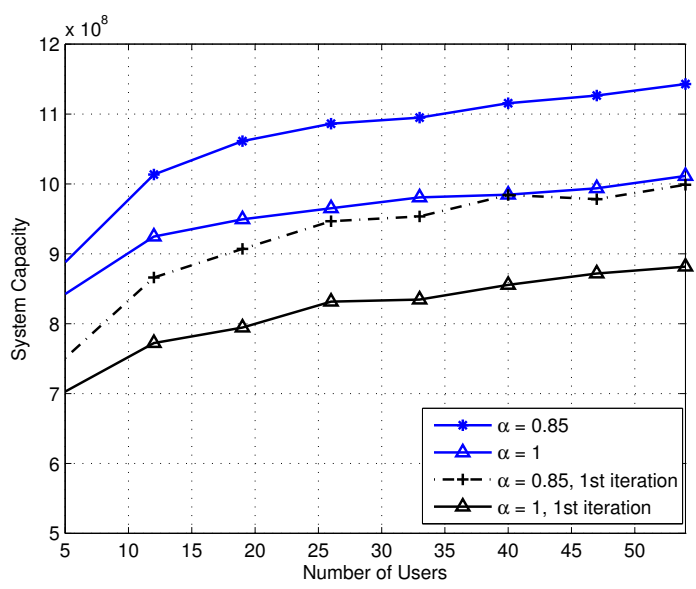

Fig. 5: System capacity against the total number of users for different values of $\alpha$.



Fig. 6: System fairness against the total number of users for different values of $\alpha$.

can enhance the system capacity rather than compromising it. This is due to the fact that in the proposed system, user $j$ receives an optical interference from 15 VLC APs while the information signal comes from only one AP. Consequently, the blockages more potentially impede the interference light rather than the intended signals. On the other hand, blocking some users from some VLC APs increases the variance of the received data rates among users, which leads to decreasing the system fairness. The figures show also how the proposed joint LB and PA algorithms can significantly improve the system capacity and fairness compared to the case where one iteration is implemented only (every user is assigned to its closest AP and each AP implements the PA only once).

\section{CONCLUSION}

In this paper, a joint LB and PA algorithms for hybrid RF/VLC network were proposed. An iterative algorithm was proposed to maximize the system capacity and enhance the system fairness. An iterative algorithm was proposed to assure that the power is allocated to the users based on the exact interference information. In the PA subproblem, we provided a new algorithm to obtain the optimal dual variables after formulating them in terms of each other. As a future work, we recommend to implement the joint power and bandwidth allocation instead of implementing the power allocation only.

\section{REFERENCES}

[1] C.-L. Tsai and Z.-F. Xu, "Line-of-sight visible light communications with InGaN-based resonant cavity LEDs," IEEE Photon. Technol. Lett., vol. 25, no. 18, pp. 1793-1796, 2013.

[2] Y. Wang, D. A. Basnayaka, and H. Haas, "Dynamic load balancing for hybrid Li-Fi and RF indoor networks," in Proc. IEEE Int. Conf. Commun. Workshop (ICCW), London, UK, 2015, pp. 1422-1427.

[3] M. Kashef, M. Abdallah, K. Qaraqe, H. Haas, and M. Uysal, "On the benefits of cooperation via power control in OFDM-based visible light communication systems," in Proc. IEEE Personal, Indoor, and Mobile Radio Commun. (PIMRC) Conf., Washington, DC, USA, Sept. 2014, pp. $856-860$.

[4] D. A. Basnayaka and H. Haas, "Hybrid RF and VLC systems: Improving user data rate performance of VLC systems," in Proc. IEEE Veh. Technol. Conf. (VTC Spring), Glasgow, UK, May 2015, pp. 1-5.

[5] Y. Wang and H. Haas, "Dynamic load balancing with handover in hybrid Li-Fi and Wi-Fi networks," J. Lightw. Technol, vol. 33, no. 22, pp. 4671-4682, Nov. 2015.

[6] M. Kashef, M. Ismail, M. Abdallah, K. A. Qaraqe, and E. Serpedin, "Energy efficient resource allocation for mixed RF/VLC heterogeneous wireless networks," IEEE J. Selec. Areas Commun., vol. 34, no. 4, pp. 883-893, 2016.

[7] X. Wu, M. Safari, and H. Haas, "Bidirectional allocation game in visible light communications," in Proc. IEEE Veh. Technol. Conf. (VTC Spring), Montreal, Canada, 2016, pp. 1-5.

[8] H. Wang, A. Liu, and X. Pan, "Optimization of joint power and bandwidth allocation in multi-spot-beam satellite communication systems," Math. Problems in Eng., vol. 2014, 2014.

[9] J. M. Kahn and J. R. Barry, "Wireless infrared communications," Proc. IEEE, vol. 85, no. 2, pp. 265-298, 1997.

[10] W. Guo, S. Wang, and X. Chu, "Capacity expression and power allocation for arbitrary modulation and coding rates," in Proc. IEEE Wireless Commun. and Netw. Conf. (WCNC), Shanghai, China, 2013, pp. 3294-3299.

[11] S. Boyd and L. Vandenberghe, Convex Optimization. Cambridge University Press, 2004.

[12] X. Li, R. Zhang, and L. Hanzo, "Cooperative load balancing in hybrid visible light communications and WiFi," IEEE Trans. Commun., vol. 63, no. 4 , pp. $1319-1329,2015$. 